\title{
Décentralisation et responsabilisation
}

\author{
Vincent Lemieux \\ Université Laval
}

Dans un chapitre de l'ouvrage sur la décentralisation gouvernementale, paru sous la direction de MarcUrbain Proulx, la décentralisation était évaluée par rapport à un certain nombre de critères, dont celui de la responsabilisation ${ }^{1}$. Nous voudrions reprendre cet exercice huit ans plus tard dans le contexte nouveau créé par le changement de gouvernement, survenu en avril 2003, pour montrer que la responsabilisation est le critère principal de la décentralisation, et que c'est la décentralisation politique qui satisfait le mieux à ce critère.

Nous montrerons également qu'il y a des obstacles à la responsabilisation et nous ferons, pour finir, quelques commentaires sur le projet de régionalisation du nouveau gouvernement libéral.

\section{Les critères d'évaluation de la décentralisation}

Les organisations décentralisées se caractérisent par leur statut, leurs compétences, leurs sources de financement et leur autorité. À chacune de ces attributions correspondent des critères qui servent à évaluer la décentralisation ${ }^{2}$.

1) Le statut des organisations décentralisées est défini principalement par leur relation au centre dont elles dépendent à des degrés divers. Dans la déconcentration, ou décentralisation administrative, les organisations administratives sur le territoire ne sont que des bureaux très dépendants de l'organisation centrale des ministères. Dans la délégation, ou décentralisation fonctionnelle, les organisations décentralisées sont des organismes autonomes qui dépendent un peu moins du centre. C'est le cas, par exemple, des régies régionales de la santé et des services sociaux. Dans la dévolution, ou décentralisation politique, les instances décentralisées sont dirigées par des élus (provinciaux, municipaux, scolaires) encore moins dépendants du centre. Enfin, dans la privatisation, ou décentralisation structurelle, les organisations décentralisées sont des entreprises totalement autonomes ou partiellement autonomes (quand elles font l'objet de la sous-traitance) par rapport au centre.

\section{La responsabilisation est le critère principal de la décentralisation, et c'est la décentralisation politique qui satisfait le mieux à ce critère.}

2) Les critères d'évaluation de la décentralisation qui découlent du statut des organisations décentralisées sont ceux de l'imputabilité et de la responsabilité. L'imputabilité consiste à rendre des comptes au centre, alors que la responsabilité consiste à répondre aux attentes de la base. La responsabilisation, quant à elle, signifie l'accroissement de responsabilité envers la base qui est entraînée par la décentralisation. On estime généralement que c'est dans la décentralisation politique que cet accroissement est le plus grand.

3) Les compétences qui sont attribuées aux organisations décentralisées renvoient à des secteurs d'activité et aux fonctions à être remplies dans ces secteurs. Les compétences des bureaux déconcentrés des ministères et des organismes autonomes sont généralement unisectorielles, comme celles des organisations privatisées. Au contraire, les organisations décentralisées dirigées par des élus ont généralement des compétences dans plusieurs secteurs d'activité (les commissions scolaires faisant exception à cet égard). L'exercice des compétences est souvent 
évalué selon le critère de l'efficacité, c'est-à-dire de l'atteinte des objectifs fixés, mais aussi selon le critère de la coordination dans la poursuite des différents objectifs. Comme nous le notions dans notre chapitre de 1995, l'efficacité et la coordination dépendent de la capacité qu'ont les organisations décentralisées de régler les problèmes dans les secteurs d'activité où elles ont des compétences. Ce qui renvoie aux deux autres attributions des organisations décentralisées, les sources de financement et l'autorité.

\section{La responsabilisation signifie I'accroissement de responsabilité envers la base qui est entraînée par la décentralisation.}

4) Les sources de financement varient selon le statut des instances décentralisées. Les bureaux déconcentrés des ministères n'ont pas de sources de financement autonomes, les organismes dits autonomes ou bien n'en ont pas ou n'ont que des sources autonomes limitées, alors que la privatisation entraîne un financement autonome. Enfin, les sources autonomes de financement sont toujours plus grandes dans la décentralisation politique que dans la décentralisation administrative ou dans la décentralisation fonctionnelle (à l'exception des entreprises publiques).

L'utilisation des sources de financement par les organisations décentralisées pour la production de biens et services est généralement évaluée selon les critères de l'efficience et de l'équité. L'efficience consiste à produire une quantité donnée de biens ou de services au plus bas coût possible, alors que l'équité consiste en des contributions ou des répartitions concernant ces biens et services qui tiennent compte des ressources et des contraintes des différentes catégories de la population. En ce sens, l'équité est un facteur d'égalisation.

Ces deux critères sont très discutés, encore plus que les quatre précédents. Certains prétendent que la décentralisation permet moins d'efficience que la centralisation, alors que d'autres pensent le contraire. Comme l'a montré Jacques Des- biens ${ }^{3}$, cela dépend des secteurs d'activité concernés. Quant à l'équité, c'est une valeur que plusieurs considèrent comme menacée par la décentralisation politique. Celle-ci créerait des inégalités dans l'accès aux biens et services dont profiteraient les localités ou les régions les mieux nanties.

5) La décentralisation comporte finalement un transfert d'autorité, qu'il s'agisse de la sélection de ceux qui dirigent les organisations décentralisées ou de l'exercice de l'autorité par ces dirigeants. Les détenteurs d'autorité dans les bureaux déconcentrés des ministères sont sélectionnés par les dirigeants de ces ministères, mais ils ont l'autorité d'appliquer des lois, règlements ou résolutions. Dans les organismes autonomes, les détenteurs d'autorité sont sélectionnés ou bien par le centre ou bien par la base, et ils peuvent adopter des résolutions, en plus d'appliquer des lois ou règlements. Il en est de même des organisations privatisées. Dans la décentralisation politique, les dirigeants sont sélectionnés par la base et l'exercice de l'autorité s'exprime par l'adoption de lois ou de règlements (dans les États fédérés), ou de règlements (dans les municipalités), avec en plus des résolutions.

\section{Les sources autonomes de financement sont toujours plus grandes dans la décentralisation politique que dans la décentralisation administrative ou dans la décentralisation fonctionnelle.}

Ces organisations ont aussi l'autorité d'appliquer certaines lois ou certains règlements adoptés par les paliers supérieurs de gouvernement. On peut évaluer la sélection des détenteurs d'autorité et l'exercice de l'autorité selon les critères de la participation et de la représentativité. La participation réfère au nombre et à la qualité de ceux qui prennent part à ces processus, alors que la représentativité réfère au caractère représentatif des participants par rapport à l'ensemble des acteurs qui sont concernés par les lois, règlements ou résolutions adoptés ou mis en œuvre. 


\section{Le caractère fondamental de la responsabilisation}

Comme nous l'avons déjà noté, c'est dans la décentralisation politique que la responsabilisation envers la base a le plus de chances de se produire. Cette responsabilisation consiste dans le fait, pour les dirigeants, de présenter des solutions qui répondent aux attentes des publics auxquels ils sont redevables des ressources qu'ils utilisent dans la production des biens et services qui sont de leur compétence. C'est surtout au moment des élections que les dirigeants doivent répondre à ces attentes. Dans la mesure où elle permet cet exercice de la responsabilité, la décentralisation politique peut être considérée comme le moyen par excellence de la responsabilisation.

Le caractère fondamental de la responsabilisation apparaît dans le fait qu'elle est sous-jacente à chacun des autres critères de la décentralisation. Ainsi, l'imputabilité des organisations décentralisées par rapport à leur centre est fondée sur la responsabilité que les dirigeants du centre éprouvent par rapport à leur base. Par exemple, si les élus municipaux demeurent imputables par rapport au ministre des Affaires municipales et au gouvernement du Québec, c'est parce que ceux-ci représentent la population du Québec dans son ensemble et sont responsables envers elle.

\section{C'est dans la décentralisation politique que la responsabilisation envers la base a le plus de chances de se produire.}

L'efficacité des organisations décentralisées est mesurée par rapport aux objectifs qui leur sont imposés ou qu'elles se donnent. En démocratie représentative, ces objectifs doivent être acceptables à la base puis être atteints le mieux possible. Il y a là un motif important de responsabilisation pour les dirigeants élus, qui se trouvent plus ou moins condamnés à l'efficacité.

Quant à la coordination, elle assure que les différentes productions de biens et services sont compatibles entre elles. On pense au développement écono- mique par rapport à la protection de l'environnement, ou encore à l'utilisation pour fins de loisirs municipaux de certains équipements scolaires. Cette coordination nécessaire repose sur la responsabilisation des dirigeants envers le public, considéré de façon globale plutôt que dans ses divisions sectorielles. À cet égard, la décentralisation politique, la seule à être vraiment multisectorielle, est un meilleur gage de coordination responsable que les autres modalités de décentralisation.

L'efficience a elle aussi son fondement dans la responsabilisation. Elle répond aux attentes de la population sur l'utilisation optimale des ressources financières transmises aux dirigeants. Ces attentes sont d'autant plus grandes qu'il y a responsabilité fiscale, c'est-à-dire que les ressources utilisées proviennent en grande partie de la base de l'organisation décentralisée, ce qui ne peut se produire qu'en décentralisation politique.

Pour ce qui est de l'équité, on peut la considérer, au même titre que la coordination, comme un critère horizontal plutôt que vertical. C'est surtout pour les grandes organisations décentralisées, comme les États fédérés, que la responsabilisation est source d'équité. Seules, elles ont les moyens de répondre aux attentes de leur population ou d'une partie d'entre elle par une certaine égalisation des ressources, alors que les organisations municipales ne peuvent aller très loin sur le chemin de l'équité.

Il apparaît évident que la responsabilisation est sousjacente à la participation. C'est parce qu'ils veulent responsabiliser leurs dirigeants, au moment des élections ou dans la prise de décision, que des citoyens participent à ces processus.

Il en est de même de la représentativité, qui ajoute à la participation le fait que des acteurs de différentes catégories sociales ou de différents milieux prennent part aux processus électoraux et aux processus de prise de décision. Ils manifestent ainsi que l'appel à la responsabilisation n'est pas monopolisé par des acteurs plus intéressés que d'autres à la composition et au fonctionnement des organisations décentralisées. 


\section{Les obstacles à la responsabilisation}

Les considérations précédentes doivent être complétées par d'autres portant sur les obstacles à la responsabilisation des dirigeants dans les organisations décentralisées. Ces obstacles n'ont pas manqué d'être signalés par les spécialistes de la décentralisation. On peut les regrouper en cinq catégories.

Premièrement, il y a l'obstacle de la dilution de la responsabilité par une sectorialisation excessive. La situation actuelle au Québec illustre bien cet obstacle avec les réseaux municipaux, scolaires et sociosanitaires qui sont décentralisés de façon parallèle, sans beaucoup de liaisons entre eux. À ces réseaux s'ajoutent des organisations décentralisées fonctionnellement, par voie de délégation, dans le secteur du développement économique, du tourisme, de l'environnement, de la culture, etc. La responsabilisation des dirigeants locaux ou régionaux s'en trouve diluée, aucune catégorie d'entre eux n'ayant à prendre charge de l'ensemble des attentes des publics.

\section{On peut se demander, au Québec, s'il ne faudrait pas opter pour la région ou la sous-région (les MRC ou les villes- centres) comme lieu principal de la décentralisation politique.}

Deuxièmement, l'étagement des organisations décentralisées, du palier supérieur au palier inférieur, peut être vu comme un autre obstacle à la responsabilisation. Si l'on considère que le gouvernement du Québec est décentralisé par rapport à celui d'Ottawa, il n'y a pas moins de quatre paliers de décentralisation sur le territoire québécois : le palier dit national, le palier régional qui correspond aux régions administratives, le palier sous-régional qui est celui des MRC et des commissions scolaires (avec un découpage territorial différent dans les deux cas), et le palier local qui est celui des municipalités locales, certaines d'entre elles correspondant à des sous-régions ou même à des régions (c'est le cas de Laval et de Montréal). Cet étagement est lui aussi une source de dispersion de la responsabilisation, même si certains font valoir qu'il a l'avantage pour les citoyens de chercher à obtenir à un palier des biens et services qu'ils ne réussissent pas à obtenir à un autre palier. De même qu'on se demande, en France, si l'on ne devrait pas opter pour la région ou pour le département comme palier principal de la décentralisation politique, on peut se demander, au Québec, s'il ne faudrait pas opter pour la région ou la sous-région (les MRC ou les villes-centres) comme lieu principal de la décentralisation politique.

Troisièmement, la responsabilisation des dirigeants des organisations décentralisées par rapport à leurs publics est aussi limitée par des interventions du centre, davantage commandées par le principe de supériorité que par le principe de serviabilité ${ }^{4}$. C'est ainsi que le gouvernement conservateur de Margaret Thatcher, en Grande-Bretagne, a réduit la capacité de taxation des municipalités les plus dépensières sous le prétexte qu'elles n'étaient pas suffisamment efficientes ${ }^{5}$, considérant en somme que c'était lui, davantage que les gouvernements municipaux en question, qui était le lieu de la responsabilisation en ce domaine.

Quatrièmement, comme il arrive souvent, ces prétentions du centre avaient en Grande-Bretagne une dimension partisane. Les municipalités les plus dépensières étaient pour la plupart tenues par les travaillistes, adversaires du gouvernement conservateur en place. De même, aux États-Unis, sous le président Reagan, le réaménagement dans les subventions accordées aux États fédérés a eu pour effet, selon Wolman, de favoriser les milieux ruraux, plus favorables au parti du président qu'à ses adversaires démocrates. Là encore, le centre à Washington se considérait comme plus responsable que les États décentralisés par rapport à la population.

\section{Le projet de régionalisation du nouveau gouvernement libéral consiste dans la création, au palier régional, de conférences régionales des élus.}

Cinquièmement, la responsabilisation est aussi limitée par une faible participation et une faible représentativité des citoyens qui s'intéressent aux processus électoraux et aux processus de prise de décision dans les organisations décentralisées. Les 
dirigeants élus peuvent alors adopter et mettre en œuvre des mesures qui répondent surtout aux attentes de leurs entourages et des groupes d'intéressés les plus puissants, sans qu'il y ait responsabilisation de la part des élus par rapport à l'ensemble des citoyens.

C'est à la lumière des considérations que nous avons faites sur la responsabilisation et sur les obstacles qu'elle comporte que nous ferons quelques réflexions, pour finir, sur le projet actuel de régionalisation du nouveau gouvernement libéral à Québec.

\section{Le projet actuel de régionalisation du gouvernement libéral}

Le projet de régionalisation du nouveau gouvernement libéral consiste dans la création, au palier régional, de conférences régionales des élus qui regrouperaient, dans chacune des régions, des élus municipaux, les préfets des MRC, les députés provinciaux et les députés fédéraux. Ces conférences remplaceraient les conseils régionaux de développement (aussi nommés conseils régionaux de concertation et de développement) et auraient, comme ces conseils, des fonctions de coordination. Au moment où nous terminons cet article, soit à la mi-août 2003, les compétences, les sources de financement et l'autorité des conférences régionales des élus restent à déterminer. Nous pouvons quand même faire quelques remarques sur ces conférences comme instruments de responsabilisation ainsi que sur les obstacles qu'elles devront surmonter à cette fin.

En principe, étant donné que les conférences seront dirigées par des élus territoriaux, elles auront plus de chances de travailler à une coordination responsable envers toute la population que ce pouvait être le cas dans les conseils régionaux, dirigés par des «nommés » de nature sectorielle. C'est du moins ce qu'on peut espérer.

Par contre, on peut se demander si la présence des députés provinciaux et fédéraux dans les conférences régionales ne comporterait pas le risque, non pas tellement de considérations partisanes, mais de préoccupations propres aux paliers supérieurs dans le fonctionnement des conférences régionales. Il serait sans doute préférable que les élus provinciaux et fédéraux forment un comité à l'intérieur des conférences régionales, qui serait consultatif par rapport à elles, au même titre que les comités sectoriels qui pourront être créés dans le secteur du développement économique, du tourisme, de l'environnement, de la culture, etc.

\section{À I'heure actuelle, les conférences régionales d'élus qui sont projetées apparaissent davantage comme un nouveau projet de régionalisation que comme un progrès sur la voie de la décentralisation politique.}

On peut s'interroger également sur la place que tiendront les élus scolaires dans les conférences régionales. Il n'y aura pas de véritable coordination régionale si elle n'inclut pas le secteur scolaire, de même d'ailleurs que le secteur socio-sanitaire. Répétons que la responsabilisation des dirigeants est plus grande quand elle est multisectorielle plutôt que découpée en secteurs. Dans le domaine scolaire comme dans le domaine socio-sanitaire, on peut souhaiter que des comités des conférences régionales se préoccupent de la coordination à opérer et des voies d'avenir de la décentralisation dans ces domaines.

À l'heure actuelle, les conférences régionales d'élus qui sont projetées apparaissent davantage comme un nouveau projet de régionalisation, plus ou moins opposé à celui du gouvernement précédent, que comme un progrès sur la voie de la décentralisation politique. Dans le mandat donné aux conférences régionales, il serait désirable qu'on leur demande de réfléchir sur l'avenir de la décentralisation politique, et en particulier sur la répartition des compétences, des sources de financement et de l'autorité entre les paliers local, sous-régional et régional, à partir de l'expérience des élus en ces matières.

Ce qui nous amène à conclure, comme nous le faisions dans notre chapitre de 1995, en disant que la décentralisation politique à venir aura plus de chances de réussir si elle s'inspire des sentiers déjà parcourus plutôt que des trottoirs dessinés d'avance par les planificateurs. 


\section{Notes et références}

1 Lemieux, V. (1995), « Participation et démocratie», in M.U. Proulx (sous la dir. de), Regards sur la décentralisation gouvernementale au Québec, Université du Québec à Chicoutimi, GRIR, p. 57-63.

2 Lemieux, V. (2001), Décentralisation, politiques publiques et relations de pouvoir, Montréal, Les Presses de l'Université de Montréal, p. 50-52.

3 Desbiens, J. (1995), «Les économies d'échelle dans les services municipaux au Québec », in M.-U. Proulx (sous la dir. de), Regards sur la décentralisation gouvernementale au Québec, Université du Québec à Chicoutimi, GRIR, p. 65-86.

4 Lemieux, V. (1997), La décentralisation, Sainte-Foy, Les Presses de l’Université Laval, p. 44-49.

5 Wolman, H. (1988), «Understanding Recent Trends in Central-local Relations: Centralisation in Great Britain and Decentralisation in United States », European Journal of Political Research, p. 425-435. 